\title{
Dielectric Study of Methyl Acrylate-Alcohol Mixtures Using Time Domain Reflectometry
}

\author{
K. Dharmalingam, ${ }^{*}$ K. Ramachandran, P. Sivagurunathan, B. Prabhakar Undre, ${ }^{\dagger}$ P. W. Khirade, ${ }^{\dagger}$ and S. C. Mehrotra ${ }^{\ddagger}$ \\ Department of Physics, Annamalai Lniversity, Anhamalai Nagar-608 002, Tamilnadu, India. E-mail: mukdhar97ârediffmail.com \\ ${ }^{\dagger}$ Department of Physics; Dr. B. A. A. Lnversity, Aurangabad-431 004, Aaharashtra, India \\ -Department of Electronics and Comptiter Science, Dr. B. A. M. University, Aurangabad - 431 004, Aaharashtra, India
}

Received September 6, 2006

\begin{abstract}
Dielectric stıdies of methyl acrylate with 1-propanol. 1-butanol. 1-heptanol and l-octanol binary mixtures have been carried out over the frequency range from $10 \mathrm{MHz}$ to $10 \mathrm{GHz}$ at temperatures of $283,293,303$ and 313 $\mathrm{K}$ using Time Domain Reflectometry (TDR) for various concentrations. The Kirkwood correlation factor and excess inverse relaxation time were determined and discussed to yield information on the molecular stnucture and dy'namics of the mixture. The values of the static dielectric constant. relaxation time and the Kirkwood correlation factor decrease with increased concentration of methyl acrylate in alcohol. The Bnggman plot shows a non-linearity of the curves for all the sy stems studied indicates the heterointeraction which may be due to hydrogen bonding of the $-\mathrm{OH}$ group of alcohol with $\mathrm{C}=\mathrm{O}$ of the methyl acrylate. The excess inverse relavation time values are negative for all the systems at all the temperatures indicates that the solute-solvent interaction hinders the rotation of the dipoles of the system.
\end{abstract}

Key Words : Dielectric relaxation, Time domain reflectometry. Alcohols

\section{Introduction}

Acry lic esters are important industrial chemicals and are widely used as precursors in the production of technically important high polymeric and latex systems. ${ }^{l}$ Dielectric relaxation studies on binary mixtures are important for understanding the hydrogen bonding and intermolecular interactions in the mixture. Alcohols play an important role in many chemical reactions due to the ability to undergo self-association with manifold internal structures and are in wide use in industry and science as reagents. solvents and fuels and attract great attention as useful solvents in the green technology: "Shirke et al..$^{3.4}$ reported the dielectric parameters for methyl acetate-alcohol and ethyl acetatealcohol mixtures at different temperatures and concentrations. Chaudhari et af. ${ }^{5}$ reported the dielectric parameters for butyl acetate-alcohol mixtures using the time domain technique. Recently our research group has investigated the complex formation of alkyl methacrylates with proton donors (primary alcohols) using FTIR spectroscopic ${ }^{6-16}$ technique.

The aim of the present investigation is to study the molecular interaction in methyl acrylate-alcohol mixtures in the microwave region $(10 \mathrm{MHz}$ to $10 \mathrm{GHz}$ ) with varying concentrations $(0 \%$ to $100 \%)$ using TDR technique for temperatures ranging from $283 \mathrm{~K}$ to $313 \mathrm{~K}$. The relaxation behaviors of these mixtures were explained by the Debye model. The excess inverse relaxation time is reported. The Kirkwood correlation factor is used to understand the molecular orientation in the mixture.

\section{Experimental}

Materials. Methyl acrylate (MA) is an Aldrich product with a manufacturer stated purity of greater than $99 \%$ on a mole basis and is used as such. MA was stabilized with about $0.002 \%$ hydroquinone monomethyl ether. AnalaR grade alcohols were purified by standard methods. ${ }^{17}$ The mixtures of various compositions $(0 \%$ to $100 \%)$ were prepared by volume before mixing.

Apparatus. The complex dielectric constant spectra were studied using time domain reflectometry. The HewlettPackard HP 54750 sampling oscilloscope with HP 54754 A TDR plug-in module was used. A fast rising step voltage pulse of about $39 \mathrm{ps}$ rise time generated by a pulse generator was propagated through a coaxial line sy'stem of characteristic impedence $50 \Omega$ Transmission line system under test was placed at the end of the coaxial line in the standard military application (SMA) coaxial cell coaxial connector with $3.5 \mathrm{~mm}$ outer diameter and $1.35 \mathrm{~mm}$ effective pin length.

All measurements were done under open load conditions. The change in the pulse after reflection from the sample placed in the cell was monitored by the sampling oscilloscope. In this experiment. a time window of 5 ns was used. The reflected pulses without sample $R_{1}(t)$ and with sample $R_{x}(t)$ were digitized in 1024 points in the memory of the oscilloscope and transferred to a PC through 1.44 MB floppy diskette drive.

A temperature controller system with a water bath and thermostat has been used to maintain the constant temperature within the accuracy limit of $\pm 1{ }^{\circ} \mathrm{C}$. The sample cell was surrounded by a heat-insulating container through which the water of constant temperature using a temperature controller system was circulated. The temperature at the cell is checked using an electronic thermometer.

Data Analysis. The time dependent data were processed to obtain complex reflection coefficient spectra $\rho^{*}(\omega)$ over 
the frequency range from $10 \mathrm{MHz}$ to $10 \mathrm{GHz}$ using Fourier transformation $^{18.13}$ as

$$
\rho^{*}(\omega)=(c / j \omega d)[p(\omega) / q(\omega)]
$$

where $p(\omega)$ and $q(\omega)$ are Fourier transforms of $\left(R_{1}(t)-R_{x}(t)\right)$ and $\left(R_{1}(t)+R_{\mathrm{x}}(t)\right)$. respectively, $c$ is the velocity of light. $\omega$ is angular frequency, and $d$ is the effective pin length and $j=$ y'-1.

The complex dielectric constant spectra $\varepsilon^{*}(\alpha)$ were obtained from reflection coefficient spectra $\rho^{*}(\omega)$ by applying a bilinear calibration method (i) $^{\text {ij }}$

The experimental values of $\varepsilon^{*}$ are fitted with the Debye equation $^{21}$

$$
\varepsilon^{*}(\omega)=\varepsilon_{\omega \phi}+\frac{\varepsilon_{0}-\varepsilon_{\omega \phi}}{1+j \omega \tau}
$$

with $\varepsilon_{i} . \varepsilon_{* 0}$. and $\tau$ as fitting parameters. A non-linear least square fit method ${ }^{2-}$ was used to determine the values of dielectric paranteters.

\section{Results and Discussion}

The determined values of static dielectric constant $\left(\varepsilon_{1}\right)$. dielectric constant at high frequency $\left(\varepsilon_{t_{0}}\right)$ and relaxation

Table 1. Dielectric Data for the Binary Mistures of Methyl

\begin{tabular}{|c|c|c|c|c|}
\hline $\begin{array}{c}\text { Volume } \% \\
\text { of MA }\end{array}$ & $\mathrm{T}=283 \mathrm{~K}$ & $\mathrm{~T}=293 \mathrm{~K}$ & $\mathrm{~T}=303 \mathrm{~K}$ & $\mathrm{~T}=313 \mathrm{~K}$ \\
\hline & & $c_{0 m}$ & & \\
\hline $100 \%$ & 7.85 & 7.41 & 6.85 & 6.55 \\
\hline $75 \%$ & 11.11 & 10.21 & 9.84 & 8.26 \\
\hline $50 \%$ & 13.31 & 12.95 & 11.81 & 10.41 \\
\hline $25 \%$ & 19.94 & 18.87 & 17.64 & 16.35 \\
\hline \multirow[t]{2}{*}{$0 \%$} & 21.89 & 20 & 18.86 & 17.34 \\
\hline & & $\varepsilon(x, m$ & & \\
\hline $100 \%$ & 2.56 & 2.45 & 2.4 & 2.34 \\
\hline $75 \%$ & 3.04 & 2.71 & 2.53 & 2.46 \\
\hline $50 \%$ & 3.09 & 2.86 & 2.65 & $2.6 \mathrm{l}$ \\
\hline $25 \%$ & 3.13 & 2.98 & 2.81 & 2.76 \\
\hline \multirow[t]{2}{*}{$0 \%$} & 3.19 & 3.05 & 2.91 & 2.89 \\
\hline & & $\tau(p \mathrm{~s})$ & & \\
\hline $100 \%$ & 12 & 8.5 & 5.3 & 2.8 \\
\hline $75 \%$ & 40.65 & 32.24 & 20.87 & 15.32 \\
\hline $50 \%$ & 102.57 & 88.75 & 64.56 & 53.85 \\
\hline $25 \%$ & 186.45 & 157.38 & 121.98 & 103.47 \\
\hline \multirow[t]{2}{*}{$0 \%$} & 402.9 & 335.1 & 252.6 & 177 \\
\hline & & $g^{+f^{\prime}}$ & & \\
\hline $100 \%$ & 0.97 & 0.94 & 0.92 & 0.89 \\
\hline $75 \%$ & 1.59 & 1.46 & 1.37 & 1.29 \\
\hline $50 \%$ & 1.84 & 1.78 & 1.69 & 1.58 \\
\hline $25 \%$ & 2.46 & 2.35 & 2.24 & 2.16 \\
\hline $0 \%$ & 2.98 & 2.83 & 2.69 & 2.47 \\
\hline
\end{tabular}
Acrylate (MA) with 1-Propanol time $(\tau)$ for solutions of different concentrations of alcolools at different temperatures are presented in Tables 1-4. The values of dielectric parameters ( $\varepsilon_{1 .} \varepsilon_{\infty}$ and $\tau$ ) for the mixtures increase towards the respective dielectric parameter of the respective alcohol for all the systems and for all the temperatures. This trend indicate the heterointeraction which may arise due to the formation of hydrogen bonding between the $-\mathrm{OH}$ group of alcohol molecules and $\mathrm{C}=\mathrm{O}$ of MA molecules. Also. it is found that in the alcohol-rich region, the dielectric parameters for the mixtures increases. whereas when the concentration of MA dominates in the mixture, the dielectric parameters decreases. Similar conclusions were drawn by Sivagurunathan et al. for methyl methacrylate -alcohols ${ }^{23}$ binary mixtures.

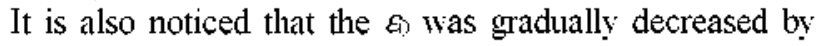
increasing the number of carbon atoms in the alkyl chain of alcohols. This trend could be attributed to the decrease in the number of dipoles in the complex, which may lead to a decrease in the molar volume of the rotated molecules. ${ }^{-4}$

The dielectric relaxation time shows continuous increase with chain length of alcohols and offers hindrance to the rotation of the molecule. The increase in relaxation time with chain length is to be expected in view of the fact that the hydroxyl group reorientation depends to some extent on

Table 2. Dielectric Data for the Binary Mixtures of Methyl

\begin{tabular}{|c|c|c|c|c|}
\hline $\begin{array}{c}\text { Volume } \% \\
\text { of } \mathrm{MA}\end{array}$ & $\mathrm{T}=283 \mathrm{~K}$ & $\mathrm{~T}=293 \mathrm{~K}$ & $\mathrm{~T}=303 \mathrm{~K}$ & $\mathrm{~T}=313 \mathrm{~K}$ \\
\hline & & $\varepsilon_{0 r r t}$ & & \\
\hline $100 \%$ & 7.85 & 7.41 & 6.85 & 6.55 \\
\hline $75 \%$ & 10.45 & 9.76 & 8.81 & 7.98 \\
\hline $50 \%$ & 12.53 & 11.64 & 10.36 & 9.64 \\
\hline $25 \%$ & 16.14 & 14.96 & 13.55 & 12.88 \\
\hline \multirow[t]{2}{*}{$0 \%$} & 17.2 & 15.97 & 14.59 & 12.72 \\
\hline & & $\varepsilon \cdots m$ & & \\
\hline $100 \%$ & 2.56 & 2.45 & 2.4 & 2.34 \\
\hline $75 \%$ & 2.85 & 2.67 & 2.48 & 2.32 \\
\hline $50 \%$ & 2.91 & 2.73 & 2.57 & 2.49 \\
\hline $25 \%$ & 2.97 & 2.85 & 2.63 & 2.56 \\
\hline \multirow[t]{2}{*}{$0 \%$} & 3.03 & 2.96 & 2.7 & 2.64 \\
\hline & & $\tau(\mathrm{ps})$ & & \\
\hline $100 \%$ & 12 & 8.5 & 5.3 & 2.8 \\
\hline $75 \%$ & 60.72 & 45.66 & 32.63 & 20.45 \\
\hline $50 \%$ & 165.23 & 131.35 & 75.39 & 61.37 \\
\hline $25 \%$ & 364.32 & 248.63 & 157.87 & 128.54 \\
\hline \multirow[t]{2}{*}{$0 \%$} & 833 & 519.8 & 342.3 & 2003 \\
\hline & & $g^{\Leftrightarrow / f}$ & & \\
\hline $100 \%$ & 0.97 & 0.94 & 0.92 & 0.89 \\
\hline $75 \%$ & 1.66 & 1.59 & 1.42 & 1.39 \\
\hline $50 \%$ & 1.95 & 1.83 & 1.78 & 1.67 \\
\hline $25 \%$ & 2.57 & 2.49 & 2.4 & 2.35 \\
\hline $0 \%$ & 2.99 & 2.85 & 2.78 & 2.68 \\
\hline
\end{tabular}
Acrylate (MA) with l-Butanol 
Table 3. Dielectric Data for the Binary Mistures of Methyl Acrylate (MA) with 1-Heptanol

\begin{tabular}{|c|c|c|c|c|}
\hline $\begin{array}{l}\text { Volume } \% \\
\text { of MA }\end{array}$ & $\mathrm{T}=283 \mathrm{~K}$ & $\mathrm{~T}=293 \mathrm{~K}$ & $\mathrm{~T}=303 \mathrm{~K}$ & $\mathrm{~T}=313 \mathrm{~K}$ \\
\hline & & $\varepsilon_{\mathrm{im} m}$ & & \\
\hline $100 \%$ & 7.85 & 7.41 & 6.85 & 6.53 \\
\hline $75 \%$ & 9.32 & 8.86 & 7.75 & 7.05 \\
\hline $50 \%$ & 11.21 & 10. 27 & 9.56 & 8.37 \\
\hline $25 \%$ & 12.02 & 11.01 & 10.12 & 9.16 \\
\hline \multirow[t]{2}{*}{$0 \%$} & 12.64 & 11.5 & 10.75 & 9.69 \\
\hline & & $c \% m$ & & \\
\hline $100 \%$ & 2.56 & 2.45 & 2.4 & 2.34 \\
\hline $75 \%$ & 2.63 & 2.58 & 2.45 & 2.36 \\
\hline $50 \%$ & 2.79 & 2.65 & 2.52 & 2.4 \\
\hline $25 \%$ & 2.86 & 2.73 & 2.59 & $2.4 \hat{3}$ \\
\hline \multirow[t]{2}{*}{$0 \%$} & 2.92 & 2.85 & 2.63 & 2.45 \\
\hline & & $\tau(\mathrm{ps})$ & & \\
\hline $100 \%$ & 12 & 8.5 & 5.3 & 2.8 \\
\hline $75 \%$ & 95.32 & 69.36 & 52.68 & 30.58 \\
\hline $50 \%$ & 328.65 & 246.57 & 129.97 & 90.63 \\
\hline $25 \%$ & 493.87 & 356.45 & 248.44 & 189.78 \\
\hline \multirow[t]{2}{*}{$0 \%$} & 1240 & 980 & 740.84 & 575 \\
\hline & & $g^{e f f}$ & & \\
\hline $100 \%$ & 0.97 & 0.94 & 0.92 & 0.89 \\
\hline $75 \%$ & 1.75 & 1.68 & 1.54 & 1.46 \\
\hline $50 \%$ & 2.18 & 2.05 & 1.95 & 1.82 \\
\hline $25 \%$ & 2.89 & 2.72 & 2.64 & 2.48 \\
\hline $0 \%$ & 3.06 & 2.96 & 2.83 & 2.76 \\
\hline
\end{tabular}

the length of the alkyl group. and the viscosity of the liquid. The relaxation time increases with increasing alkyl chain length. which indicates that the degree of cooperativity for reorientation of the molecules increases with increasing length and the bulk of the cluster increases. The relaxation time increases with increasing chain length in agreement with the fact that the relaxation time is directly related to the size of the molecules. 5,36

It was pointed out by Smyth ${ }^{37}$ that the relaxation time of a proton donor must increase as the donor ability of the donor solute. Our results are in accordance with this conclusion. It is also noticed that. the $\tau$ value of mixtures lie between the individual component values. this result further confinns the existence of solute-solvent interaction between the alcohol and MA molecules.

The relaxation time decreases with increase in temperature from 283 to $313 \mathrm{~K}$ for all the molecules under investigation. This may possibly be due to the decrease in the molar volume and the increase in the effective length of the dipole with increase in temperature and also due to increase in the thermal agitation and partly due to decrease in the viscosity $\mathrm{s}^{\circ}$

The information related to the solute-solvent interaction is also given by the Bruggman factor. The effective volume of
Table 4. Dielectric Data for the Binary Mixtures of Methyl Acrylate (MA) with l-Octanol

\begin{tabular}{|c|c|c|c|c|}
\hline $\begin{array}{l}\text { Volume } \% \\
\text { of MA }\end{array}$ & $\mathrm{T}=283 \mathrm{~K}$ & $\mathrm{~T}=293 \mathrm{~K}$ & $\mathrm{~T}=303 \mathrm{~K}$ & $\mathrm{~T}=313 \mathrm{~K}$ \\
\hline & & $\varepsilon_{0 r r t}$ & & \\
\hline $100 \%$ & 7.85 & 7.41 & 6.85 & 6.55 \\
\hline $75 \%$ & 8.95 & 8.19 & 7.88 & 6.97 \\
\hline $50 \%$ & 10.04 & 8.98 & 8.07 & 7.16 \\
\hline $25 \%$ & 10.87 & 9.74 & 8.97 & 7.98 \\
\hline \multirow[t]{2}{*}{$0 \%$} & 11.24 & 10.08 & 9.21 & 8.19 \\
\hline & & $\varepsilon_{\cdots, m}$ & & \\
\hline $100 \%$ & 2.56 & 2.45 & 2.4 & 2.34 \\
\hline $75 \%$ & 2.47 & 2.38 & 2.29 & 2.21 \\
\hline $50 \%$ & 2.32 & 2.26 & 2.17 & 2.11 \\
\hline $25 \%$ & 2.18 & 2.12 & 2.08 & 2.01 \\
\hline \multirow[t]{2}{*}{$0 \%$} & 2.07 & 2.03 & 1.98 & 1.94 \\
\hline & & $\tau(\mathrm{ps})$ & & \\
\hline $100 \%$ & 12 & 8.5 & 5.3 & 2.8 \\
\hline $75 \%$ & 94.21 & 82.14 & 60.37 & 49.87 \\
\hline $50 \%$ & 395.65 & 286.32 & 156.35 & 122.46 \\
\hline $25 \%$ & 587.24 & 398.76 & 278.55 & 201.28 \\
\hline \multirow[t]{2}{*}{$0 \%$} & 1356.4 & 1085.6 & 862.19 & 675.3 \\
\hline & & $g^{e f f}$ & & \\
\hline $100 \%$ & 0.97 & 0.94 & 0.92 & 0.89 \\
\hline $75 \%$ & 1.86 & 1.73 & 1.62 & 1.55 \\
\hline $50 \%$ & 2.29 & 2.15 & 2.01 & 1.93 \\
\hline $25 \%$ & 2.96 & 2.83 & 2.74 & 2.61 \\
\hline $0 \%$ & 3.15 & 3.08 & 2.99 & 2.84 \\
\hline
\end{tabular}

the solute gets modified by solute-solvent interactions and is best illustrated by the non-linearity of Bruggeman formula ${ }^{\text {ig }}$

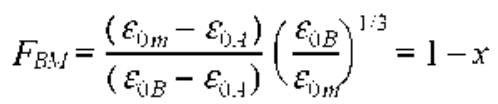

The Bruggeman plots of volume fraction of alcohol versus $F_{B, i}$ for methyl acrylate-alcohols mixtures are given in Figure 1. It can be seen from these figures that $F_{B i t}$ is not a linear function of volume fraction of alcohol. The nonlinearity of the curves indicates heterointeraction which may be due to hydrogen bonding of the $-\mathrm{OH}$ group of alcohol with $\mathrm{C}=\mathrm{O}$ of the $\mathrm{MA}^{-33}$

The structural information about the liquids from the dielectric relaxation parameter may be obtained using the Kirkwood correlation parameter $g .^{3 j}$ This factor is also a parameter for obtaining information regarding orientation of electric dipoles in polar liquids. The $\mathrm{g}$ for the pure liquid may be obtained using the expression

$$
\frac{4 \pi N \mu^{2} \rho}{9 k T} g=\frac{\left(\varepsilon_{01}-\varepsilon_{\infty \mathrm{s}}\right)\left(2 \varepsilon_{01}+\varepsilon_{\mathrm{se}}\right)}{\varepsilon_{01}\left(\varepsilon_{\infty}+2\right)^{2}}
$$

where $\mu$ is the dipole moment in the gas phase. $\rho$ is the density at temperature T.M is the molecular weight. $k$ is the 

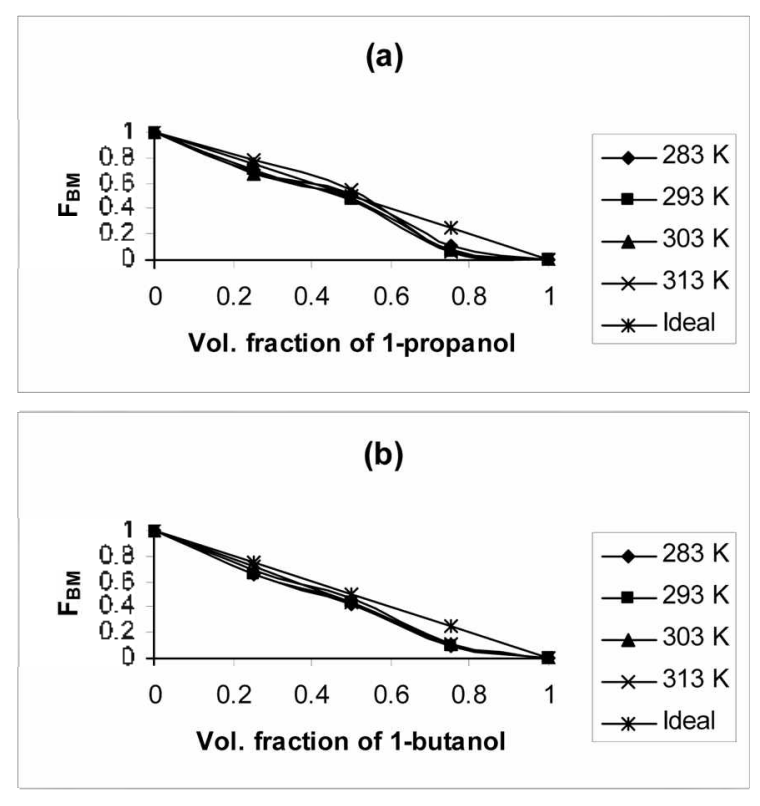

(c)

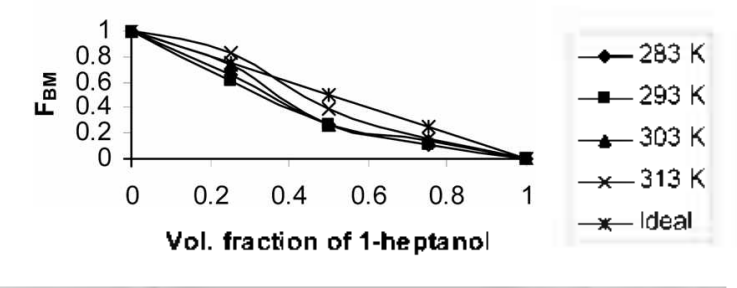

(d)

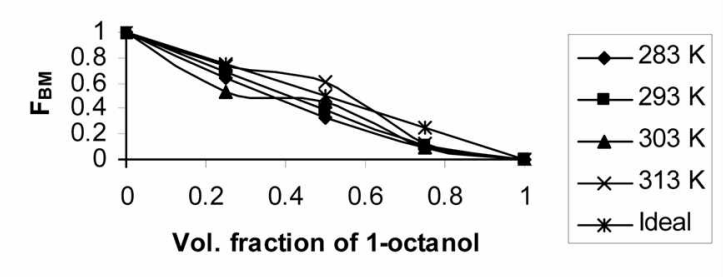

Figure 1. Bruggman plot for (a) MA-1-propanol, (b) MA-Ibutanol, (c) MA-1-heptanol and (d) MA-1-octanol mixtures.

Boltzmann constant, and $N$ is Avogadro's number.

A modified form of this equation ${ }^{31}$ has been used to study the orientation of electric dipoles in binary mixtures. For the mixtures of two polar liquids. say A and B. equation (4) is modified by assuming that $g^{g}$ has become the effective correlation factor in the mixture. The Kirkwood equation for the mixture may be expressed as using the following assumption:

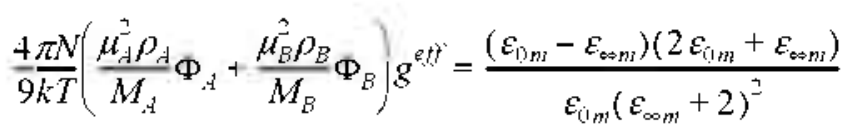

with $\phi_{A}$ and $\phi_{B}$ as the volume fractions of liquids $A$ and $B$. respectively. In equation (5) the values of $g^{2 f f}$ will change from $g_{A}$ to $g_{B}$ as the concentration of molecule $B$ is increased from $0 \%$ to $100 \%$.

The calculated values of $g^{\text {tff }}$ using Eq. (4) for all the syistems at different concentrations at different temperatures are given in Tables 1-4. The effective value of the correlation factor $g^{2 f}$ for methyl acrylate is smaller than the corresponding $g^{\text {eff }}$ values for different alcohols. The value of $g^{2 t}$ for pure methyl acrylate is close to unity indicating no dipole correlation and the $g^{\text {et }}$ values for pure alcohols are greater than unity indicating a high degree of coordinated chainlike structures. For the mixtures of alcohols with methyl acrylate. the values of $g^{2 \text { th }}$ show an increasing trend with the increasing concentration of alcohol in all the mixture. This trend suggests reorientation of neigliborhood molecules of the constituent polar liquids forming a tendency towards parallel alignment of dipoles. This conclusion is similar to that arrived from the variation of dielectric parameters with concentration. The Kirkwood correlation factor is found to be an increasing function of the size of the alkyl group of alcohols. This is probably due to the fact that when the alkyl group is small the polar groups are relatively exposed. so that dipolar interactions may occur between associated complexes which tend to counteract the coordination within the complexes. ${ }^{32}$

At high concentrations of alcohol in the mixtures, there are a large number of alcohol molecules surrounding the MA molecules. The associative alcohol molecules act as proton donors enabling hydrogen bonding with the MA molecules. Thus dipole-dipole interaction occurs in such a way that the effective dipole moment gets increased and linear $\alpha$-multimers are fonned. ${ }^{28}$ The dipole-dipole interaction is the interaction of the $-\mathrm{OH}$ group of alcohol with $\mathrm{C}=\mathrm{O}$ of MA.

At low concentrations of alcohol in the mixtures there are only a small number of alcohol molecules to enable dipoledipole interactions through hydrogen bonding with the nonassociative MA molecules. As a result. weak intermolecular interaction occurs.

The information related to dynamics of the solute-solvent interaction may be obtained by excess properties ${ }^{33}$ related to the relaxation time in the mixture. The excess inverse relaxation time is defined as

$$
(1 / \tau)^{E}=(1 / \tau)_{m}-\left[\left((1 / \tau)_{.1} x_{.1}+(1 / \tau)_{B} x_{B}\right)\right]
$$

where $(1 / \tau)^{E}$ is the excess inverse relaxation time which represents the average broading of dielectric spectra. The information regarding the dynamics of solute-solvent interaction from this excess property is as follows:

(i) $(1 / \tau)^{\prime \prime}=0$ : There is no change in the dynamics of solute-solvent interaction.

(ii) $(1 / \tau)^{n}<0$ : The solute-solvent interaction produces a field such that the effective dipoles rotate slowly:

(iii) $(1 / \tau)^{h}>0$. The solute-solvent interaction produces a field such that the effective dipoles rotate quickly i.e.. the field co-operate in the rotation of the dipoles.

The variation of $(1 / \tau)$ with volume fraction of MA at temperatures 283, 293. 303 and $313 \mathrm{~K}$ is shown in Figure 2. 

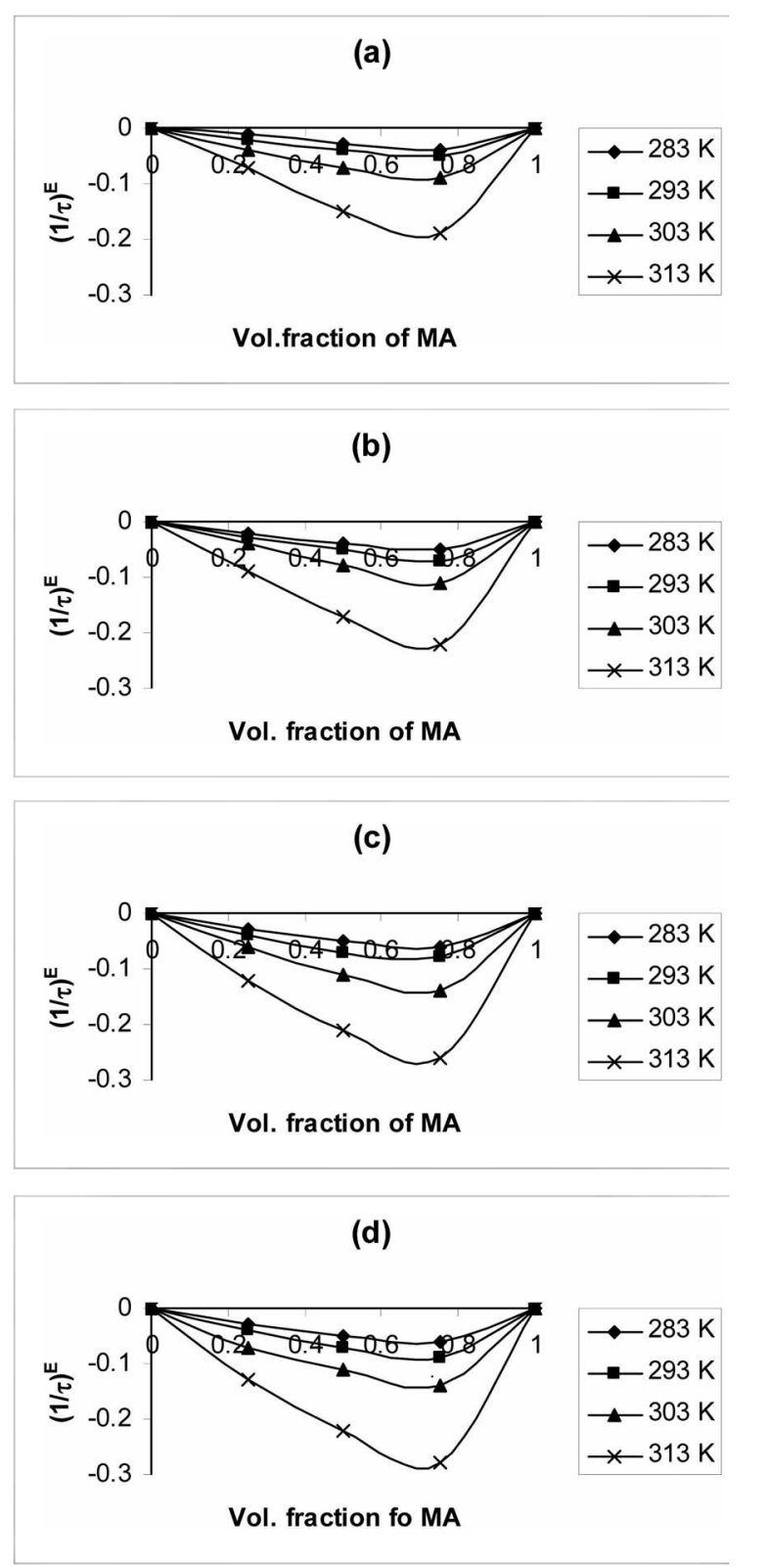

Figure 2. Excess inverse relaxation time versus mole fraction of MA for (a) MA-l-propanol, (b) MA-1-butanol, (c) MA-1-heptanol and (d) MA-l-octanol mixtures.

The excess inverse relaxation time values are negative for all concentrations and all temperatures and all the systems studied indicates slower rotation of the dipoles. due to the solute-solvent interaction form the hy'drogen bonded structures which produces a field in such a way that the effective dipole rotation is hindered.

\section{Conclusions}

A dielectric relaxation study on methyl acrylate-alcohol binary mixtures has been carried out at different concentrations and four temperatures using Time Domain Reflectometry (TDR). The values of dielectric parameters show systematic change with increase in concentration. temperature and alkyl chain length of alcohols. The negative excess inverse relaxation time values indicate the slower rotation of effective dipoles of the system. These data provide infonmation regarding solute-solvent interaction in liquids

\section{References}

1. Schildknecht. C. E. Innt and Related Polnters: Wiley: New York. 1977.

2. Savage. P. E. Chem Rev $1999,99.603$.

3. Shirke. R. M.; Chaudhari, A.: More, N. M.: Patil. P. B. J. Chem. Eng. Data 2000. $45,917$.

4. Shirke. R. M.: Chaudhari, A.; More, N. M.: Patil, P. B. J. Mol Liq. 2001. 94. 27.

5. Chaudhari. A.: Shirke. R. M.: More. N. M.: Patil. P. B. J. Sol. Chen 2002. 31.305

6. Sivagurunathan, P; Dharmalingam. K; Ramachandran, K. Z. Phys. Chem. 2005, 219. 1385.

7. Sivagurunathan, P: Dhammalingam, K.: Ramachandran. K. Spectrochint Acta 2006. 64.4. 127.

8. Sivagurunathan. P.: Dharmalingam. K.: Ramachandran. K. Z. Phis Chem. 2005. 219. 1635.

9. Sivagurunathan. P; Dharmalingam. K: Ramachandran, K. Indian J. Pure \& Appl Plys 2005. 43.905

10. Sivagurunathan. P.: Dhamalingam. K.: Ramachandran, K. Ham Group Chemistry 2005. 4.241.

11. Sivagurunathan. P.: Dharmalingam. K.: Ramachandranı. K. Indian J. Phis 2005. 79. 1403 .

12. Dharmalingam. K:: Ramachandrant. K.: Siqagunulnathat1. P. Spectrochin Acta 2007. 66.1, 48.

13. Dharmalingam, K.; Ramachandran. K. Plns. Cham Liq. 2006, 44, 77.

14. Dharmalingam. K: Ramachandran. K.: Sivagurunathan, P. Z. Phs Chen. 2006. 220.739.

15. Dharmalingam, $K$; Ramachandran, K; Sivagurunathan. P. S. Afr: J. Chem. 2006, 59.75 .

16. Sivagurunathan. P.: Dharmalingam, K.; Ramachandran. K. J. Sol. Chem $2006,35,1467$.

17. Vogal, A. I. Text Book of Practical Organic Chemistru $3^{\text {rd }}$ ed: Longmant: London. 1957.

18. Samulon. H. A. Proc. IRE. 1951. 39, 175.

19. Shan11on. C. E. Proc. IRE. 1949.37. 10.

20. Cole. R. H.; Berbarian, J. G.; Mashimo. S.; Chryssitios, G.: Burns, A: Tombari, E. J. Appl. Ploss. 1989. 66,793.

21. Debye. P. Polar Holecules: Chemical Catalog: New York. 1929.

22. Bevington. P. R. Data Reduction and Error Analysis for the Phwsical Sciences. Mc Graw-Hill: New York. 1969.

23. Sivagurunathan. P.: Dharmalingam. K: Ramachandrant. K.: Prabhakar Undre. B.: Khirade. P. W: Mehrotra, S. C. Main Group Chemistry 2005. 4, 235 .

24. Dhamalingan, K.: Ramachandran. K.: Sivagurunathan. P.: Kalanse, G. M. Hain Group Chemisny 2005. 4. 227.

25. Sivagurunathan. P.: Dharmalingam. K.: Ramachandrant. K.: Prabhakar Undre. B.: Khirade. P. W.: Mehrotra. S. C. Phil. Mag. Lett. 2006, 86. 291

26. Dharmalingam. K.; Ramachandran, K.: Sivagurunathan. P: Prabhakar Undre. B.: Khirade, P. W: Mehrotra. S. C. 1 fol Plons. 2006, 104. 2835.

27. Smyth. C. P. In Moleculor Interactions: Ratajczak. H.. OrvilleThomas. W. T.. Eds.: Tohn Wiley and Sons: New York. 1981: Vol. II.

28. Sivagurunathan. P.: Dharmalingam. K.: Ramachandran. K.: Prabhakar Undre. B.: Khirade, P. W: Mehrotra, S. C. Plysica B 2007, 387. 201.

29. Bruggeman. D. A. G. Am. Plys. 1935. 5.636.

30. Kirkwood. T. G. J. Chem. Phis. 1939. 7.911.

31. Kumbharkhane. A. C.: Puranik. S. M.: Mehrotra. S. C. J. Sol. Chen 1993. 22.219.

32. Pieruecini, M.: Saija. F. J. Chem. Plys. 2004. 121,3191.

33. Chaudhari. A.: Mehrotra. S. C. Hol Phus. 2002. 100.3907. 